\title{
Developing Optimized Trajectories Derived from Mission and Thermo-structural Constraints
}

\author{
Matthew H. Lear, ${ }^{*}$ Brian E. McGrath, ${ }^{\dagger}$ Michael P. Anderson,${ }^{\ddagger}$ and Peter W. Green ${ }^{\S}$ \\ The Johns Hopkins University Applied Physics Laboratory, Laurel, Maryland, 20723, USA
}

In conjunction with NASA and the Department of Defense, the Johns Hopkins University Applied Physics Laboratory (JHU/APL) has been investigating analytical techniques to address many of the fundamental issues associated with solar exploration spacecraft and high-speed atmospheric vehicle systems. These issues include: thermo-structural response including the effects of thermal management via the use of surface optical properties for high-temperature composite structures; aerodynamics with the effects of non-equilibrium chemistry and gas radiation; and aero-thermodynamics with the effects of material ablation for a wide range of thermal protection system (TPS) materials. The need exists to integrate these discrete tools into a common framework that enables the investigation of interdisciplinary interactions (including analysis tool, applied load, and environment uncertainties) to provide high fidelity solutions.

In addition to developing robust tools for the coupling of aerodynamically induced thermal and mechanical loads, JHU/APL has been studying the optimal design of high-speed vehicles as a function of their trajectory. Under traditional design methodology the optimization of system level mission parameters such as range and time of flight is performed independently of the optimization for thermal and mechanical constraints such as stress and temperature. A truly optimal trajectory should optimize over the entire range of mission and thermo-mechanical constraints.

Under this research, a framework for the robust analysis of high-speed spacecraft and atmospheric vehicle systems has been developed. It has been built around a generic, loosely coupled framework such that a variety of readily available analysis tools can be used. The methodology immediately addresses many of the current analysis inadequacies and allows for future extension in order to handle more complex problems.

\section{Introduction}

In order to perform a full transient aero-thermo-mechanical trajectory analysis three different physicsbased models must be coupled: a six degree-of-freedom (6DOF) kinematics model, a computational fluid dynamics (CFD) model, and a computational structural mechanics (CSM) model. A range of coupling technologies are available and must be evaluated on a per-application basis. Direct coupling involves solving a complete, fully coupled set of equations that simultaneously solve for all of the necessary fluid and structural field variables. This type of coupling is computationally expensive since the most limiting time and length scales will drive the simulation and the equations must be solved over a very large domain which encompasses both the vehicle and the surrounding fluid. There are currently no commercial applications of this type so solutions using this method will require substantial code development and be limited, at least in the nearterm, by a lack of robustness. An alternative to co-solution is a server-client architecture where the 6DOF, CFD, and CSM codes are linked through a central server which manages the translation of data across the simulation boundaries. Mesh-based Parallel Code Coupling Interface (MpCCI) developed by FraunhoferInstitute SCAI is a widely supported server-client coupling tool which advertises an open architecture; however, the server and the application programming interface (API) are complex and necessitate a high

\footnotetext{
*Sr. Mechanical Engineer, Global Engagement Department, 11100 Johns Hopkins Rd, and Senior Member.

${ }^{\dagger}$ Sr. Aerospace Engineer, Global Engagement Department, 11100 Johns Hopkins Rd, and Senior Member.

${ }^{\ddagger}$ Sr. Aerospace Engineer, Global Engagement Department, 11100 Johns Hopkins Rd, and Senior Member.

$\S$ Sr. Mechanical Engineer, Air and Missile Defense Department, 11100 Johns Hopkins Rd, and Senior Member.
} 
degree of mastery of not only each coupled code but also of the server tool itself. Recently, several of the commercial codes that actively supported the MpCCI interface have begun developing their own tightly coupled CFD-CSM capabilities. A third option is loose coupling which allows interpolation of data from a CFD solution to a CSM application based upon a trajectory computed from a 6DOF simulation. In this scheme the individual codes are coupled but in a uni-directional fashion. Thus, fluid dynamic pressures and fluxes depend upon the CSM surface temperatures and the 6DOF generated flight conditions, but geometry and mass property changes from the thermo-mechanical solution do not affect the CFD or 6DOF solutions. For a given application, the degree of coupling necessary to obtain solutions of a desired fidelity needs to be evaluated; however, for the applications of interest in this research loose coupling is believed to be reasonably accurate and substantially more efficient. Thus, a loose coupling technique has been employed. A functional diagram of the loose coupling procedure is shown in Figure 1

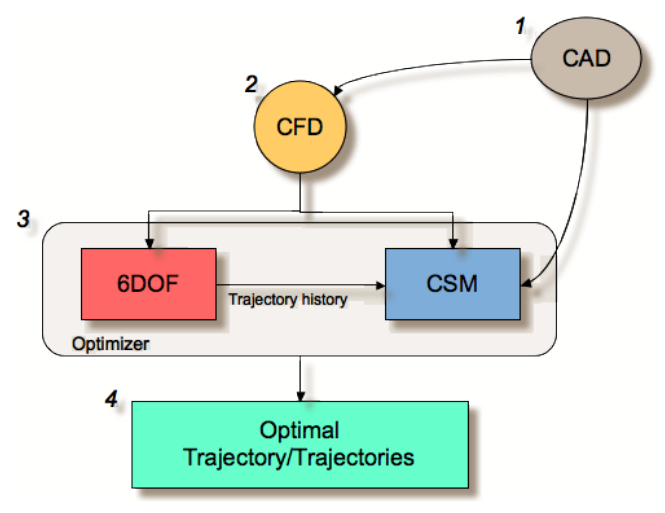

Figure 1. Functional diagram of the loose coupling process.

where the tasks required to perform the optimized trajectory analysis are labeled sequentially and described below. Currently, this methodology is restricted to using the user subroutines available in ABAQUS; however, it could easily be extended to other CSM codes as long as they have similar functionality.

1. CAD: Based upon the current or desired design a computer aided design (CAD) model must be created. The same CAD model should be used for both the CFD and CSM calculations. As will be explained in following section, the CFD and CSM meshes do not need to be continuous along the shared boundary, but in order to insure that the forces are transferred properly, it is extremely important that the solid model boundaries coincide.

2. CFD: Because this method is loosely coupled it is not necessary to recompute the CFD results during the simulation. Thus, the CFD data can be pre-computed and stored in a database that is queried as a function of the current vehicle flight conditions. For this work, the database may be a function of the altitude, total angle-of-attack, aerodynamic roll angle, and Mach number. The total angle-of-attack and aerodynamic roll angles are described in Figure 2. where $u, v$, and $w$ are the vehicle body axes.

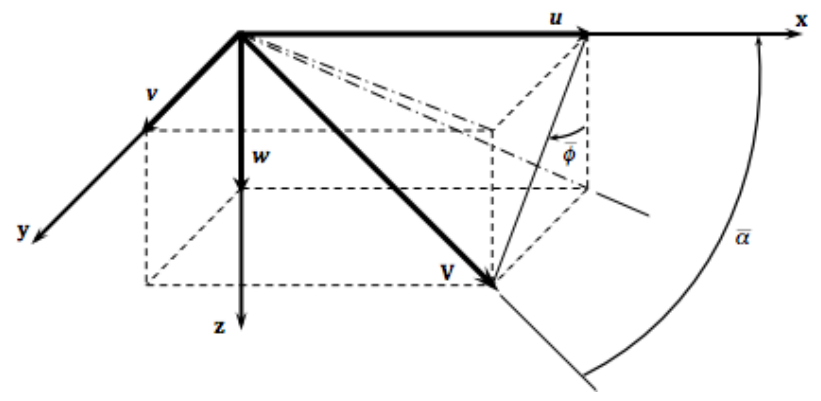

Figure 2. Aerodynamic Angle Definitions.

3. Optimizer: The 6DOF and the CSM codes are coupled within the optimizer step. The 6DOF reads force and moment coefficients from the pre-computed CFD analysis and then based upon a set of initial 
conditions propagates the vehicle in time and space until a predetermined time or position is reached. The CSM code reads the time history data from the 6DOF and using the CFD database determines the appropriate mechanical (pressure) and thermal (flux) boundary conditions as a function of time along the trajectory. Prior to launching the simulation optimization variables and corresponding thresholds are established. After the CSM analysis is complete for a given trajectory the thresholds are checked by the optimizer and the initial conditions are iterated until a converged solution is achieved.

4. Optimal Trajectories: Ideally, after a small number of iterations a trajectory optimized on a set of predetermined conditions will be found.

The results in this paper were obtained using CFD ++ as the CFD code and ABAQUS as the CSM code. The $6 \mathrm{DOF}$ and the Optimizer were developed in-house.

\section{Mesh Interpolation}

The CFD and CSM solvers need not and, in most cases, should not have matching boundary grids along the fluid-structure interface. It is, therefore, necessary to develop an interpolation scheme to translate the CFD data to the CSM grid. The concept of a kernel approximation for randomly distributed particles that has been actively developed in support of the meshless Galerkin and particle-based computational methods is used to perform the interpolation. According to this concept, a corrected kernel approximation $\tilde{f}(x)$ to a variable $f(x)$ at a point $x$ is given by (1)

$$
\tilde{f}\left(x_{i}\right)=\frac{\int f\left(x_{i}\right) W\left(x_{i}\right) d \Omega}{\int W\left(x_{i}\right) d \Omega} .
$$

If a particle approximation of Equation (1) is formed then the new particle-based corrected kernel approximation of $f(x)$ is,

$$
\tilde{\tilde{f}}\left(x_{i}\right)=\frac{\sum_{\alpha=1}^{N} f\left(x_{i}^{\alpha}\right) W^{\alpha}\left(x_{i}\right) \triangle \Omega^{\alpha}}{\sum_{\alpha=1}^{N} W^{\alpha}\left(x_{i}\right) \triangle \Omega^{\alpha}}
$$

where subscript $\alpha$ identifies the particle at $x_{i}^{\alpha}$ on the surface for which $f\left(x_{i}^{\alpha}\right)$ is known and $x_{i}$ is the point where the interpolated value is desired. In this analysis, $x_{i}^{\alpha}$ is a point on the CFD boundary and $x_{i}$ is an integration point on the CSM boundary. $W^{\alpha}\left(x_{i}\right)$ is the value of the kernel or weight from particle $\alpha$ at point $x_{i}$ and $\triangle \Omega$ is a volume associated with particle $\alpha$. Since the CFD boundary particles are not associated with any structural volume, $\triangle \Omega^{\alpha}$ is assumed to be constant for all $\alpha$ and, thus, (2) becomes the standard weighted average given below.

$$
\tilde{\tilde{f}}\left(x_{i}\right)=\frac{\sum_{\alpha=1}^{N} f\left(x_{i}^{\alpha}\right) W^{\alpha}\left(x_{i}\right)}{\sum_{\alpha=1}^{N} W^{\alpha}\left(x_{i}\right)}
$$

A variety of forms of the kernel function are possible and a piecewise cubic function was chosen ${ }^{2}$

$$
W^{\alpha}\left(\left\|x_{i}^{\alpha}-x_{i}\right\|, h\right)=\frac{1}{4 \pi h^{3}} \begin{cases}(2-\psi)^{3}-4(1-\psi)^{3} & 0 \leq \psi \leq 1 \\ (2-\psi)^{3} & 1<\psi \leq 2 \\ 0 & \psi>2\end{cases}
$$

where $\psi=\frac{\left\|x_{i}^{\alpha}-x_{i}\right\|}{h} . h$ is the kernel smoothing length and determines the extent of the influence of a given particle $\alpha$. The method of computing $h$ is largely arbitrary; however, it should not be so large that the interpolation loses its local character nor should it be so small that there are an insufficient number of particles to provide an accurate interpolation. For this analysis, $h$ was chosen such that the influence domain of a given particle was a sphere with radius equal to 1.5 times the distance to the nearest neighbor particle. ${ }^{1}$ 


\section{Time Interpolation}

Prior to starting the analysis a CFD database of pressures $(p)$, temperatures $(\theta)$, and fluxes $(q)$ along the boundary must be computed at discrete values of altitude $(h)$, total angle-of-attack $(\bar{\alpha})$, aerodynamic roll angle $(\bar{\phi})$, and Mach number $(M)$ for both an adiabatic and a cold-wall condition along the fluid-structural boundary. The cold-wall temperatures specified in the cold-wall CFD analysis should be reasonable and in the same range expected from the trajectory simulation. For the cases studied in this report sea-level ambient temperature provided good results. At the beginning and end of each computation step a three variable interpolation is performed as a function of $\bar{\alpha}, \bar{\phi}$, and $M$ using an eight node three-dimensional interpolation. A second interpolation is then performed to find the data at the correct altitude, $h$. During the CSM analysis step the current value of $p, \theta$, and $q$ will be linearly interpolated using the values from the beginning and the end of the step. More detail on this process is provided in the following derivation.

At any point on the trajectory it is assumed that the proper conditions may be approximated by the interpolation,

$$
\begin{aligned}
\xi^{1}(\bar{\alpha}, \bar{\phi}, M) & =N_{i}^{1}(\bar{\alpha}, \bar{\phi}, M) \xi_{i} \\
\xi^{2}(\bar{\alpha}, \bar{\phi}, M) & =N_{i}^{2}(\bar{\alpha}, \bar{\phi}, M) \xi_{i}
\end{aligned}
$$

where the superscripts indicate the nearest CFD database altitude above and below the current actual altitude, $\xi$ is a generic variable that represents any of the database values (i.e. $h, q, \theta$ ), and repeated indices imply summation. The shape functions, $N_{i}$, are determined using the standard tri-linear approximation given in (7).

$$
\xi(\bar{\alpha}, \bar{\phi}, M)=a_{0}+a_{1} \bar{\alpha}+a_{2} \bar{\phi}+a_{3} M+a_{4} \bar{\alpha} \bar{\phi}+a_{5} \bar{\alpha} M+a_{6} \bar{\phi} M+a_{7} \bar{\alpha} \bar{\phi} M
$$

A linear interpolation between the two database altitudes as given in Equation (8) produces the the proper interpolated value for $\xi$ at the given flight conditions.

$$
\xi\left(h, \xi^{1}, \xi^{2}\right)=\delta \xi^{2}+(1-\delta) \xi^{1}
$$

$\delta$ is a non-dimensional measure of the current altitude as given by,

$$
\delta=\left(h-h^{1}\right) /\left(h^{2}-h^{1}\right) .
$$

A final linear interpolation between the two time steps, $t_{n}$ and $t_{n+1}$, then determines the correct values of $p$, $\theta$, and $q$ at any time over the time step. A graphical representation of the CSM analysis process is shown in Figure 3.

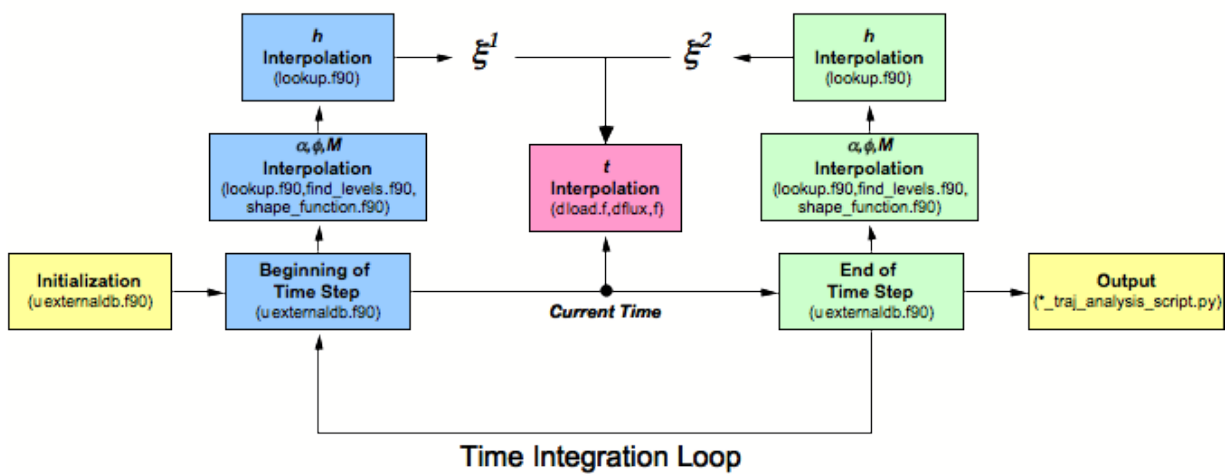

Figure 3. CSM Time Integration Procedure Functional Diagram.

\section{Aero-thermal Heating}

Convective heat transfer through the boundary layer is calculated using the pre-computed CFD adiabatic and isothermal cold-wall data. The convective heating relation is estimated using the wall temperature from 
the adiabatic analysis, $\theta_{a w}$, as the driving force. ${ }^{3}$ This, when combined with the heat flux from the isothermal cold-wall analysis, provides the convection coefficient, $\mu$, as in (10).

$$
\mu=\frac{q_{w}}{\theta_{w}-\theta_{a w}}
$$

Subscript $w$ indicates values taken at the boundary (wall) and subscript $a$ indicates results derived from the adiabatic analysis. The convective heat flux is then found through (11).

$$
q_{\text {convective }}=\mu\left(\theta_{a w}-\theta_{w}\right)
$$

The radiative heat flux may also be applied using the standard radiative heat equation given in (12).

$$
q_{\text {radiative }}=\sigma \varepsilon\left(\theta_{\infty}^{4}-\theta_{w}^{4}\right)
$$

In (10) and (12), $\mu, q_{w}$, and $\theta_{a w}$ are derived from the CFD results, $\theta_{w}$ is found from the CSM solution, and $\theta_{\infty}$ is a function of the trajectory as determined by the $6 \mathrm{DOF}$. It is also important to note that both the ambient wall temperature and the heating coefficient are interpolated to the CSM integration points using the previously described time accurate interpolation.

\section{Test Case}

The analysis methodology was applied to a simple axisymmetric ballistic re-entry vehicle. A schematic of the vehicle is given in Figure 4.

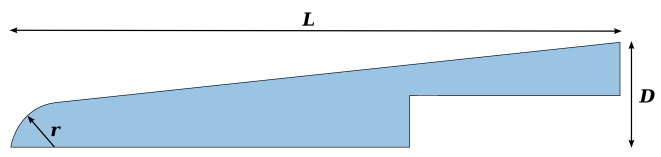

Figure 4. Axisymmetric example case geometry (not to scale).

The vehicle is solid tungsten with the following material properties:

$$
\begin{aligned}
& \rho=19,300 \mathrm{~kg} / \mathrm{m}^{3} \\
& c_{p}=132.0 \mathrm{~J} / \mathrm{kgK} \\
& k=174.0 \mathrm{~W} / \mathrm{mK} \\
& \varepsilon=0.7
\end{aligned}
$$

where $\rho$ is the mass density, $c_{p}$ is the specific heat, $k$ is the thermal conductivity, and $\varepsilon$ is the emissivity. The total angle-of-attack and the aerodynamic roll angles were zero throughout the trajectory so that the CFD database was solely a function of altitude and Mach number.

\section{V.A. Aerothermal Heating}

A complete ballistic trajectory analysis was performed and compared to results using heating correlations. ${ }^{4}$ For this simple geometry the correlations should be accurate and are, therefore, considered a reasonable source for validation. The trajectory altitude and Mach number as a function of time are given in Figure 5.

The temperatures and stagnation point heat flux are given in Figures 6 and 7, respectively, where the data identified as "Lear, Green" was generated using the current methodology and "Detra, Kemp, Riddell" was generated using the correlations. The discrete jumps in the heat flux are a result of assuming sea-level ambient conditions at all altitudes in the CFD database. Even with this approximation, the results show an excellent agreement with the heating correlations. 


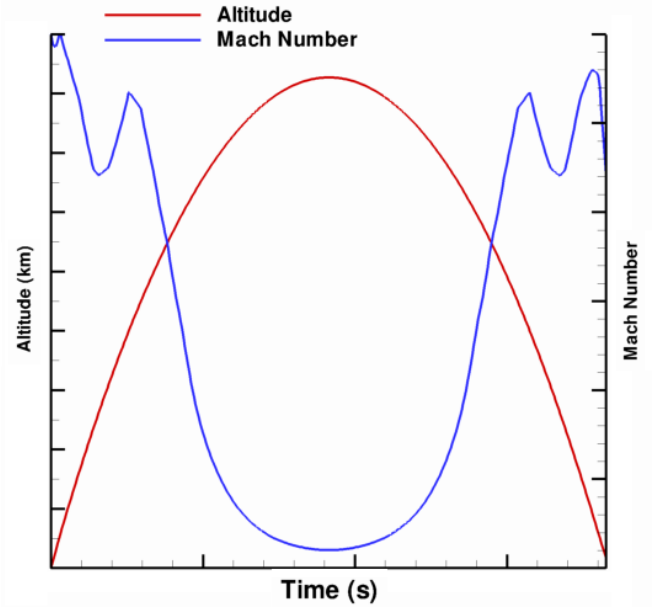

Figure 5. Example Case Trajectory Altitude and Mach Number.

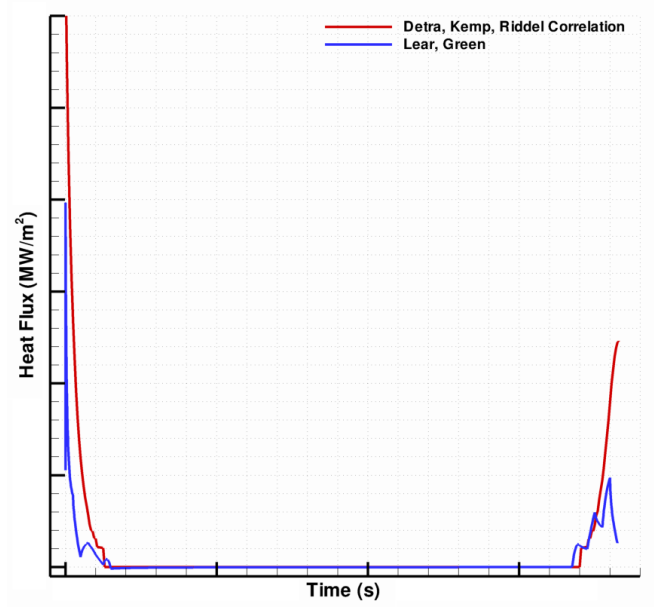

Figure 6. Stagnation Point Heat Flux.

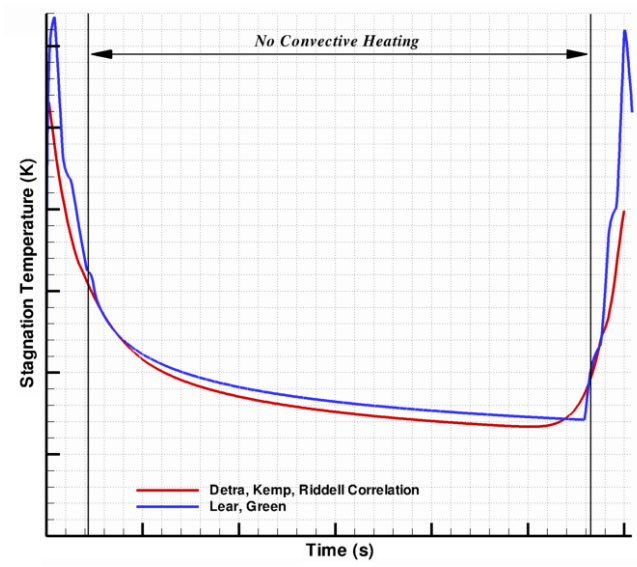

Figure 7. Stagnation Point Temperature. 


\section{V.B. Optimization}

A simplified, single variable optimization study was conducted. For this analysis a zero angle-of-attack trajectory neglecting gravitational accelerations and using sea-level flight conditions was performed. A desired stagnation point temperature was specified and initial velocity was varied using a simple NewtonRaphson scheme. The final optimal initial velocity was found to be less than $85 \%$ of the initial velocity. The analysis converged in 5 iterations and a plot of the optimization process is given in Figure 8.

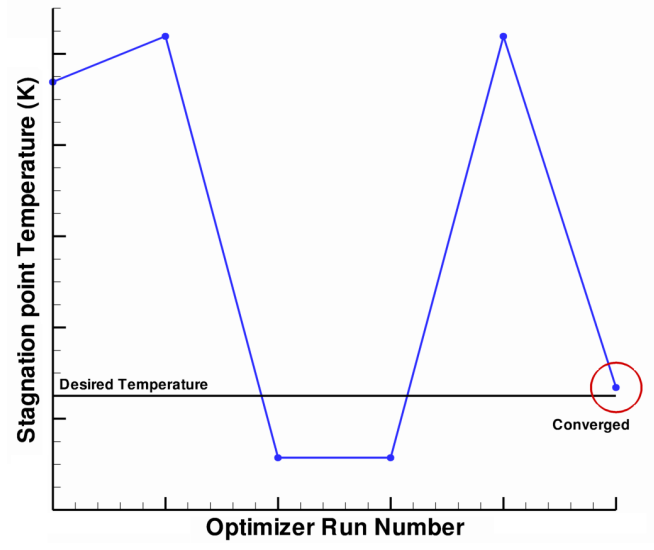

Figure 8. Optimization Progress Chart for the Example Case.

\section{Conclusion}

This research demonstrates that a robust loosely coupled aero-thermomechanical analysis methodology can be used with existing commercial codes. This allows the most flexibility in the design process because it exploits the robustness of existing codes and the availability of existing code-specific expertise. Additionally, this research has shown that this tool can be wrapped within an optimization layer in order to develop vehicle trajectories that are optimized on a complete set of variables whether they be performance or structurally based. Because of the modular nature of the methodology it is easily extendable to include other physical effects and to solve more complex problems.

\section{Acknowledgments}

The authors would like to thank Kim Bey at NASA Langley, the sponsor of this work.

\section{References}

\footnotetext{
${ }^{1}$ Liu G. and Liu M., Smoothed Particle Hydrodynamics, World Scientific, 2007

${ }^{2}$ Monaghan J. and Lattanzio J., "A Refined Particle Method for Astrophysical Problems", Astronomy and Astrophysics, 1985

${ }^{3}$ Burmeister, L., Convective Heat Transfer, John Wiley \& Sons, 1993

${ }^{4}$ Detra R., Kemp N., and Riddell F., "Addendum to Heat transfer to Satellite Vehicles Reentering the Atmosphere", Jet Propulsion, 1957

${ }^{5}$ Press W., et al., Numerical Recipes in Fortran 77, Cambridge University Press, 1992
} 